\title{
X-RAY EVOLUTION OF PULSAR WIND NEBULAE
}

\author{
Aya Bamba ${ }^{1,2}$, Takayasu Anada ${ }^{2}$, Tadayasu Dotani ${ }^{2}$, Koji Mori $^{3}$, Ryo Yamazaki $^{4}$, Ken Ebisawa $^{2}$, and Jacco Vink $^{5}$ \\ ${ }^{1}$ School of Cosmic Physics, Dublin Institute for Advanced Studies, 31 Fitzwilliam Place, Dublin 2, Republic of Ireland; abamba@cp.dias.ie \\ ${ }^{2}$ ISAS/JAXA Department of High Energy Astrophysics, 3-1-1 Yoshinodai, Chuo-ku, Sagamihara, Kanagawa 252-5210, Japan \\ ${ }^{3}$ Department of Applied Physics, Faculty of Engineering University of Miyazaki, 1-1 Gakuen Kibana-dai Nishi, Miyazaki 889-2192, Japan \\ ${ }^{4}$ Department of Physics and Mathmatics, Aoyama Gakuin University, 5-10-1 Fuchinobe, Sagamihara, Kanagawa 252-5258, Japan \\ ${ }^{5}$ Astronomical Institute, Utrecht University, P.O. Box 80000, 3508TA Utrecht, The Netherlands \\ Received 2010 June 14; accepted 2010 July 12; published 2010 July 27
}

\begin{abstract}
During the search for counterparts of very high energy gamma-ray sources, we serendipitously discovered large, extended, low surface brightness emission from pulsar wind nebulae (PWNe) around pulsars with the ages up to $\sim 100 \mathrm{kyr}$, a discovery made possible by the low and stable background of the Suzaku X-ray satellite. A systematic study of a sample of eight of these PWNe, together with Chandra data sets, has revealed that the nebulae keep expanding up to $\sim 100 \mathrm{kyr}$, although the timescale of the synchrotron X-ray emission is only $\sim 60 \mathrm{yr}$ for typical magnetic fields of $100 \mu \mathrm{G}$. Our result suggests that the accelerated electrons up to $\sim 80 \mathrm{TeV}$ can escape from the PWNe without losing most energies. Moreover, in order to explain the observed correlation between the X-ray size and the pulsar spin-down age, the magnetic field strength in the PWNe must decrease with time.
\end{abstract}

Key words: pulsars: general - stars: neutron - X-rays: ISM

\section{INTRODUCTION}

Pulsar wind nebulae (PWNe) consist of electrons and positrons accelerated by the pulsar wind termination shocks, giving rise to bright $\mathrm{X}$-ray synchrotron emission from young PWNe with ages up to several kyr. Recent X-ray observations have shown the presence of the extended PWNe (e.g., Camilo et al. 2004; Romani et al. 2005; Van Etten et al. 2008) from the pulsars with the age up to $\sim 10 \mathrm{kyr}$. However, we still do not know how the PWNe evolve when they become older than the energy-loss timescales of electrons, and whether the accelerated electrons can escape the PWNe or not. Understanding the electron/positron escape process is important, since pulsars and PWNe may be an important source of cosmic ray electrons and positrons (Kawanaka et al. 2010), as recently suggested by PAMELA (Adriani et al. 2009), ATIC (Chang et al. 2008), PPBBETS (Torii et al. 2008), Fermi (Abdo et al. 2009), and H.E.S.S. (Aharonian et al. 2009).

Recently, the Galactic plane survey by H.E.S.S. Cherenkov telescopes has revealed about 50 very high energy (VHE) gamma-ray sources (Aharonian et al. 2005a, 2006, 2008). The search for the counterparts in other wavelengths is ongoing. In fact, about a half of such VHE gamma-ray sources without any counterparts are now categorized as PWNe, ${ }^{6}$ because possibly associated old X-ray pulsars are found later. Hence, these sources are keys to understand the PWN evolution and how the accelerated particles escape from the system.

However, the problem is that such old $\mathrm{PWNe}$ are rather faint in X-rays as opposed to in VHE gamma-rays (Mattana et al. 2009). As the PWN ages, the X-ray emission becomes rapidly fainter, while the VHE gamma-ray luminosity keeps constant. Previous X-ray observations did not have enough sensitivity to detect the probable faint nebulae. We thus need deep $\mathrm{X}$-ray observations with low background to understand their $\mathrm{X}$-ray properties. The X-ray Imaging Spectrometer (XIS; Koyama et al. 2007) on board Suzaku (Mitsuda et al. 2007) has carried out deep follow-ups for these sources to search for

\footnotetext{
6 See http://tevcat.uchicago.edu/.
}

possible X-ray counterparts. Actually, Uchiyama et al. (2009) found a largely extended nebula from PSR B1823-13 associated with HESS J1825-137, which could not be detected with Chandra (Pavlov et al. 2008) or XMM-Newton (Gaensler et al. 2003) observations. PSR J1809-1917 is also in the same case with Chandra (Kargaltsev \& Pavlov 2007) and Suzaku (Anada et al. 2010). In this Letter, we will make a systematic study of X-ray structure of such old PWNe.

\section{SAMPLE SELECTION AND RESULTS}

The XIS has a low and stable background and is ideal for observing faint and extended sources like our targets. We selected the XIS deep observations of the three PWNe with VHE gamma-ray counterparts (HESS J1825-137, HESS J1809-193, and HESS J1718-385) and pulsars with known characteristic age $\left(t_{c}=P /(2 \dot{P})\right.$, with $P$ and $\dot{P}$ period and period derivative, respectively). For comparison, we also added a sample of five younger pulsars with VHE gamma-rays that have been observed with Chandra. Vela is not used in this Letter although it is a well-known PWN with $\mathrm{TeV}$ emission, since it is too extended to cover by one or a few pointings of current X-ray observatories. Table 1 lists the observational details and physical parameters of our samples, which cover most of the PWNe with known periods and period derivatives, distances, and archival deep X-ray observations. Our sample set covers the PWN candidates with ages in the range of $\sim$ kyr up to $\sim 100 \mathrm{kyr}$.

Figure 1 shows $\mathrm{X}$-ray images above $2 \mathrm{keV}$ of our sample: we did not use the photons below $2 \mathrm{keV}$ to avoid possible contamination of thermal X-rays from the surrounding supernova remnants (SNRs). All images are correct for exposure and vignetting. As can be seen, for the whole sample low surface brightness X-ray emission is detected from an extended region. This is the first discovery of low surface brightness, extended $\mathrm{X}$-ray emission from PWNe as old as $100 \mathrm{kyr}$. In order to measure the extent systematically, we made projected profiles using the rectangular regions shown in Figure 1 and fitted these with a one-dimensional (1D) Gaussian plus a constant background model to derive the typical size of the region, using the sigma 

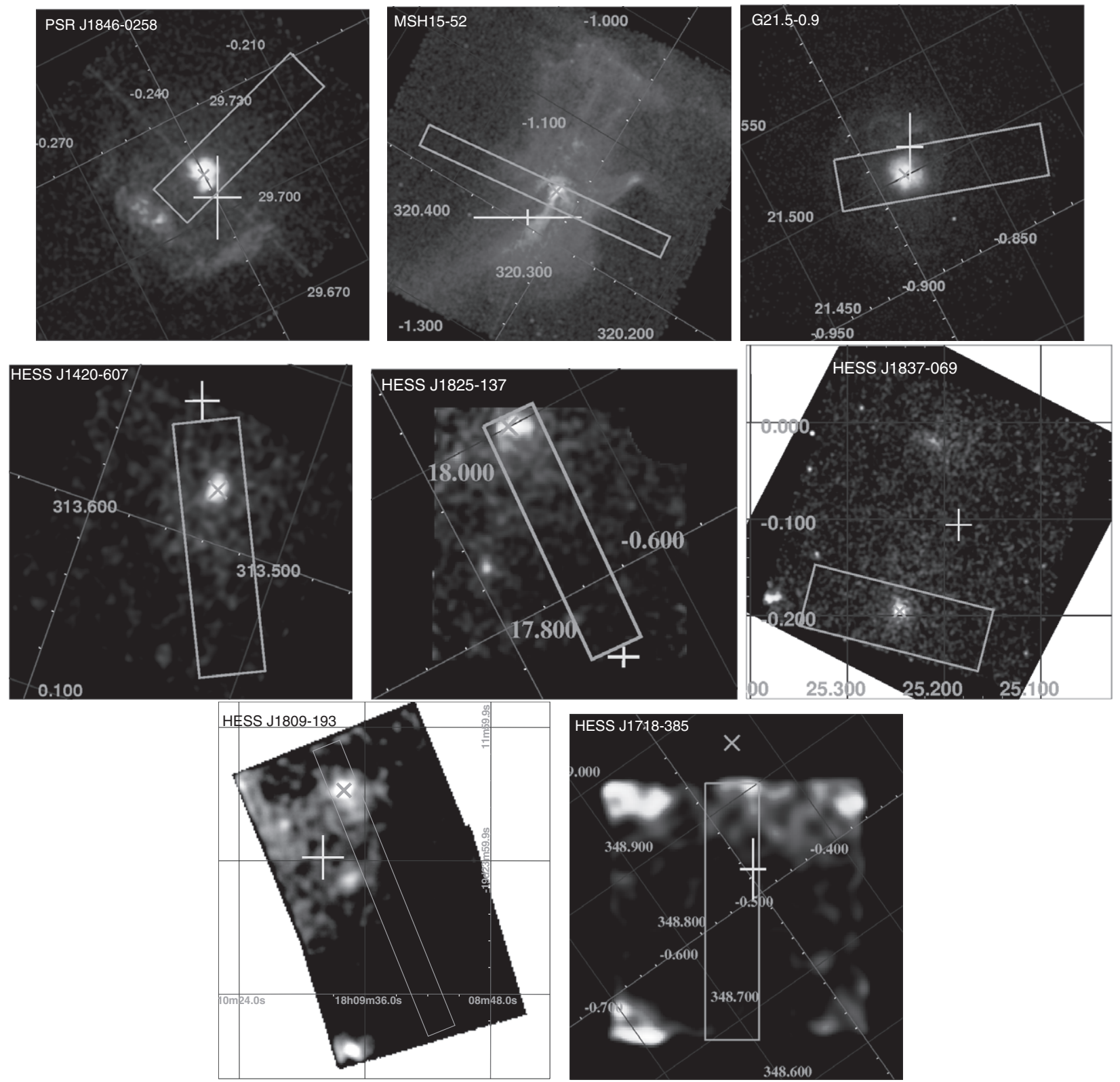

Figure 1. X-ray images of the old PWNe above $2 \mathrm{keV}$ in Galactic coordinates. The rectangles represent the region for the size estimation. The "+" and "X" marks are the positions of VHE gamma-ray emission peaks and the associated pulsars, respectively. The exposure and vignetting are corrected.

Table 1

Observational Journals and Physical Parameters for PWNe with VHE Emission

\begin{tabular}{lccccccc}
\hline \hline H.E.S.S. Name & PWN/Pulsar & Satellite & $\begin{array}{c}t_{c}{ }^{\mathrm{a}} \\
(\mathrm{kyr})\end{array}$ & $\begin{array}{c}\text { Distance } \\
(\mathrm{kpc})\end{array}$ & $\begin{array}{c}\sigma_{X} \\
(\mathrm{arcmin})\end{array}$ & $\begin{array}{c}\text { Size } \\
(\mathrm{pc})\end{array}$ & References \\
\hline HESS J1846-029 & PSR J1846-0258 & Chandra & 0.7 & $6.3 \pm 1.2$ & $0.63 \pm 0.05$ & $3.5 \pm 1.0$ & $(1),(2),(3)$ \\
HESS J1514-591 & MSH 15-52 & Chandra & 1.6 & $4.2 \pm 0.8$ & $1.6 \pm 0.1$ & $5.9 \pm 1.6$ & $(4),(5),(6)$ \\
HESS J1833-105 & G21.5-0.9 & Chandra & 4.9 & $4.3 \pm 0.9$ & $0.72 \pm 0.04$ & $2.7 \pm 0.8$ & $(4),(2),(7)$ \\
HESS J1420-607 & PSR J1420-6049 & Chandra & 13 & $5.6 \pm 1.1$ & $1.5 \pm 0.4$ & $7.3 \pm 3.8$ & $(8),(4),(9)$ \\
HESS J1825-137 & PSR B1823-13 & Suzaku & 21 & $3.9 \pm 0.8$ & $3.2 \pm 0.2$ & $10.9 \pm 3.0$ & $(4),(8),(10)$ \\
HESS J1837-069 & AX J1838.0-0655 & Chandra & 23 & $6.6 \pm 0.9$ & $1.25 \pm 0.05$ & $7.2 \pm 1.0$ & $(11),(12),(13)$ \\
HESS J1809-193 & PSR J1809-1917 & Suzaku & 51 & $3.5 \pm 0.7$ & $6.8 \pm 1.0$ & $21 \pm 8$ & $(4),(14),(15),(16)$ \\
HESS J1718-385 & PSR J1718-3825 & Suzaku & 90 & $3.6 \pm 0.7$ & $4.2 \pm 0.5$ & $13 \pm 5$ & $(4),(14)$ \\
\hline
\end{tabular}

Notes.

a The characteristic age of the pulsars.

${ }^{\mathrm{b}}$ We assumed that the center of the nebula is on the PSR J1718-3825.

References. (1) Leahy \& Tian 2008; (2) Djannati-Ată et al. 2008; (3) Helfand et al. 2003; (4) Cordes \& Lazio 2002; (5) Aharonian et al. 2005b; (6) Gaensler et al. 2002; (7) Slane et al. 2000; (8) Aharonian et al. 2006; (9) Roberts et al. 1999; (10) Uchiyama et al. 2009; (11) Davies et al. 2008; (12) Gotthelf \& Halpern 2008; (13) Anada et al. 2009; (14) Aharonian et al. 2007; (15) Anada et al. 2010; (16) Bamba et al. 2003. 

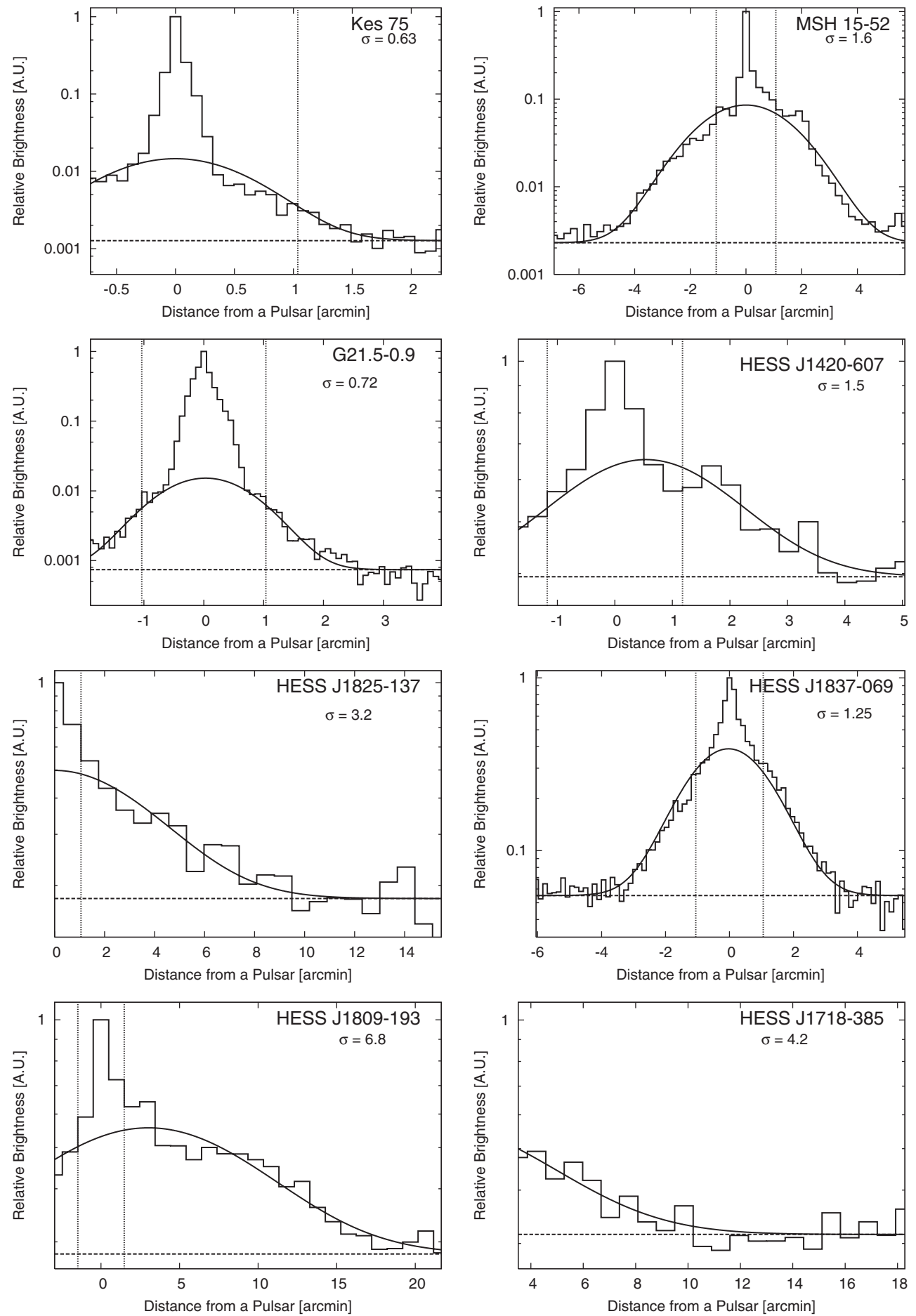

Figure 2. One-dimensional profiles of the PWNe. The solid lines represent the best-fit Gaussian model, whereas the dashed lines are the best-fit constant background. For the fitting, the pulsar region (surrounded by dotted lines) was not used.

of the Gaussian $\left(\sigma_{X}\right)$. We did not use the central pulsar region from the fitting to avoid their influence. As shown in Figure 2, a Gaussian profile provides a reasonable approximation for the surface brightness profiles. We defined the source size as three times of the $\sigma_{X}$ in the Gaussian fit. This is the value given in Table 1. The best-fit parameters converted to the physical size are also shown in Table 1. The errors include both the statistical errors as given by the fits and the distance uncertainties.

Figure 3 shows the correlation between the characteristic ages of the host pulsars and the X-ray sizes of the PWNe. It shows that the extent of the X-ray emission increases with $t_{c}$. The correlation coefficient is 0.72 . The PWNe are not spherical, and as a result the derived sizes depend on the direction of profiles. However, the uncertainty is smaller than one order, and the correlation cannot be removed with this effect. If we assume that the PWN age is equal to $t_{c}$, Figure 3 indicates that the X-ray emitting regions of PWNe keep expanding up to $\sim 100$ kyr. PSR B1706-44 is recently detected in VHE gammarays (Hoppe et al. 2009), and it has largely extended X-ray emission (Romani et al. 2005). However, no X-ray observation 


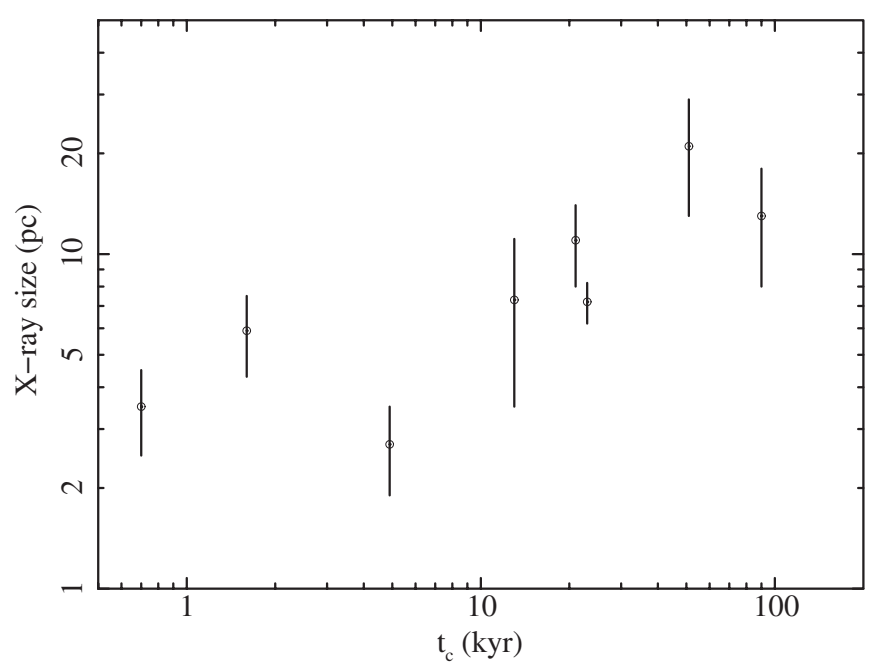

Figure 3. Correlation between the characteristic age of the pulsar, $t_{c}$, and the $\mathrm{X}$-ray physical size of the PWNe above $2 \mathrm{keV}$. Errors include both the statistical error and the distance uncertainty. It shows evolution of the emission region size in the X-ray band.

covered the entire emission, and we could not measure the extension. There are some other PWNe with known large extended X-ray nebulae, such as PSR J1016-5857 (Camilo et al. 2004) and PSR J2021+3651 (Van Etten et al. 2008), from which no detection is reported in the VHE gamma-ray band. A rough estimation of their extent and comparing it to their pulsar characteristic ages are consistent with the correlation that we find, even though these pulsars are younger than those in our sample. However, the sizes of these PWNe are somewhat more uncertain, since these PWNe are not fully covered by current $\mathrm{X}$-ray observations. Nevertheless, it suggests that the correlation between PWNe X-ray extent and characteristic ages is not only valid for VHE gamma-ray emitting PWNe, but also for PWNe in general.

\section{DISCUSSION}

We serendipitously found, for the first time, that the X-ray sizes of the PWNe keep increasing up to $\sim 100 \mathrm{kyr}$. In this section, we consider the origin of X-ray evolution of PWNe.

The energy-loss timescale of the electrons emitting synchrotron X-rays with characteristic energy $\epsilon_{\text {syn }}$ can be estimated as

$$
2\left(\frac{B}{10 \mu \mathrm{G}}\right)^{-3 / 2}\left(\frac{\epsilon_{\mathrm{syn}}}{1 \mathrm{keV}}\right)^{-1 / 2} \mathrm{kyr},
$$

where $B$ is the magnetic field strength. In the case of young PWNe, such as Crab and Vela, the magnetic field strength may be around $100 \mu \mathrm{G}$ - the equipartition magnetic field strength for these PWNe is measured to be 60-300 $\mu \mathrm{G}$ (Marsden et al. 1984; Hillas et al. 1998; de Jager et al. 1996). Suppose that the $\mathrm{X}$-ray emitting electrons are supplied by the wind termination shock, which has the radius of much less than $\sim 1 \mathrm{pc}$ (Bamba et al. 2010). Then, if the old PWNe had a similar magnetic field strength of about $100 \mu \mathrm{G}$, their X-ray sizes would be much smaller than the observed because the synchrotron cooling timescale of such electrons would be much smaller than $\sim \mathrm{kyr}$. Therefore, in order to explain the observed correlation between the X-ray size and $t_{c}$, the magnetic field strength inside the PWNe must decrease with time.

If the average magnetic field strength decays below $1 \mu \mathrm{G}$ for old PWNe, the electrons can survive enough to emit synchrotron
X-rays with a largely extended feature of $\sim 20-30$ pc. Typically, the older is a pulsar, the smaller is its spin-down luminosity. Simultaneously the magnetic field in the PWNe may decrease, as the magnetic field at the light cylinder decreases with time, or because the expansion of the PWNe dilutes the magnetic field, as the pulsar wind expands. Alternatively, if the electron/positron energy density decreases with time due to adiabatic expansion, and/or synchrotron cooling, and if the magnetic field remains in rough equipartition with the particle energy density, one also expects a decrease of the magnetic field with time.

According to the model of Kennel \& Coroniti (1984), the electrons in PWNe are advected outward. The advection speed depends on the $\sigma$ parameter, which is defined as the relative energy flux of magnetic field to the kinetic energy flux. For larger $\sigma$, the advection speed is larger, and the electrons can escape faster. In the case of young PWNe such as Crab and MSH $15-52, \sigma$ is estimated to be very small, $\sim 0.003-0.03$ (Kennel \& Coroniti 1984; Mori 2002; Gaensler et al. 2002; Yatsu et al. 2009), although Mori et al. (2004) suggested larger value $(\sigma=0.01-0.13)$. The largely extended nebula can be achieved if $\sigma$ becomes one order of magnitude larger when the system becomes $\sim 100$ kyr old.

So far we only considered that the growth of the PWNe is caused by advection from the expanding electron/positron plasma. However, one should also consider the possibility of electron/positron transport by diffusion. For the diffusion constant $D$ one can assume $D=\eta c E /(3 e B)$, with $\eta \geqslant 1$, a parameter showing deviations from Bohm diffusion $(\eta=$ 1). One can eliminate $E$ by substituting the typical relation between synchrotron photon energy and electron energy $\epsilon_{\mathrm{syn}}=$ $7.4 E^{2} B \mathrm{keV}$. These can be substituted in the expression for the diffusion length, $l_{d}$, at time $t: l_{d}=\sqrt{2 D t}$. This gives the required magnetic field, given $l_{d}, t$, and $\eta$,

$$
B=\left(\frac{\epsilon_{\mathrm{syn}}}{7.4 \mathrm{keV}}\right)^{1 / 3}\left(\eta \frac{2}{3} \frac{c}{e} t\right)^{2 / 3} l_{d}^{-4 / 3}
$$

This equation shows that in order for a PWN to reach a size of $13 \mathrm{pc}$ in X-ray due to particle diffusion, a magnetic field of $9 \mu \mathrm{G}$ (42 $\mu \mathrm{G})$ for $\eta=1(\eta=10)$ is required. In principle, the time, $t$, can be smaller than the age of the pulsar, which would correspond to a recent diffusion of the electrons, whereas the oldest electrons have already escaped. The estimated magnetic field strengths are therefore upper limits. These values are not unreasonable, but a more thorough study is required to definitely decide whether the large X-ray extents of the PWNe in our sample are due to diffusion or advection. Our result suggests that the accelerated electrons and positrons on the termination shocks can escape from the host PWN systems keeping with enough energy to emit synchrotron X-rays. Together with the fact that the majority of VHE gamma-ray unidentified sources are newly discovered old PWNe, they could be the main contributor of cosmic ray electrons and positrons.

Finally, we point out that the large extents of the PWNe of ages up to $100 \mathrm{kyr}$ are also surprising, because PWNe in SNRs should be crushed by the reverse shock (e.g., van der Swaluw 2003), whereas our result shows no evidence for an influence by the reverse shock.

We thank the anonymous referee for his/her suggestions. We acknowledge all the Suzaku team members for their gracious supports. The authors also thank D. Khangulyan for his comments. This work was supported in part by Grant-in-Aid for 
Scientific Research of the Japanese Ministry of Education, Culture, Sports, Science and Technology, no. 22684012 (A.B.) and no. 21740184 (R.Y.).

\section{REFERENCES}

Abdo, A. A., et al. 2009, Phys. Rev. Lett., 102, 181101

Adriani, O., et al. 2009, Nature, 458, 607

Aharonian, F., et al. 2005a, Science, 307, 1938

Aharonian, F., et al. 2005b, A\&A, 435, L17

Aharonian, F., et al. 2006, ApJ, 636, 777

Aharonian, F., et al. 2007, A\&A, 472, 489

Aharonian, F., et al. 2008, A\&A, 477, 353

Aharonian, F., et al. 2009, A\&A, 508, 56

Anada, T., Bamba, A., Ebisawa, K., \& Dotani, T. 2010, PASJ, 62, 179

Anada, T., Ebisawa, K., Dotani, T., \& Bamba, A. 2009, PASJ, 61, S183

Bamba, A., Mori, K., \& Shibata, S. 2010, ApJ, 709, 507

Bamba, A., Ueno, M., Koyama, K., \& Yamauchi, S. 2003, ApJ, 589, 253

Camilo, F., Gaensler, B. M., Gotthelf, E. V., Halpern, J. P., \& Manchester, R. N. 2004, ApJ, 616, 1118

Chang, J., et al. 2008, Nature, 456, 362

Cordes, J. M., \& Lazio, T. J. W. 2002, arXiv:astro-ph/0207156

Davies, B., Figer, D. F., Law, C. J., Kudritzki, R.-P., Najarro, F., Herrero, A., \& MacKenty, J. W. 2008, ApJ, 676, 1016

de Jager, O. C., Harding, A. K., \& Strickman, M. S. 1996, ApJ, 460, 729

Djannati-Ată, A., et al. 2008, Proc 30th ICRC (Mérida), 2, 823

Gaensler, B. M., Arons, J., Kaspi, V. M., Pivovaroff, M. J., Kawai, N., \& Tamura, K. 2002, ApJ, 569, 878

Gaensler, B. M., Schulz, N. S., Kaspi, V. M., Pivovaroff, M. J., \& Becker, W. E. 2003, ApJ, 588, 441
Gotthelf, E. V., \& Halpern, J. P. 2008, ApJ, 681, 515

Helfand, D. J., Collins, B. F., \& Gotthelf, E. V. 2003, ApJ, 582, 783

Hillas, A. M., et al. 1998, ApJ, 503, 744

Hoppe, S., de Oña-Wilhemi, E., Khélifi, B., Chaves, R. C. G., de Jager, O. C., Stegmann, C., \& Terrier, R., \& for the H.E.S.S. Collaboration 2009, Proc ICRC, submitted (arXiv:0906.5574)

Kargaltsev, O., \& Pavlov, G. G. 2007, ApJ, 670, 655

Kawanaka, N., Ioka, K., \& Nojiri, M. M. 2010, ApJ, 710, 958

Kennel, C. F., \& Coroniti, F. V. 1984, ApJ, 283, 694

Koyama, K., et al. 2007, PASJ, 59, 23

Leahy, D. A., \& Tian, W. W. 2008, A\&A, 480, L25

Marsden, P. L., Gillett, F. C., Jennings, R. E., Emerson, J. P., de Jong, T., \& Olnon, F. M. 1984, ApJ, 278, L29

Mattana, F., et al. 2009, ApJ, 694, 12

Mitsuda, K., et al. 2007, PASJ, 59, S1

Mori, K. 2002, PhD thesis, Osaka Univ.

Mori, K., Burrows, D. N., Hester, J. J., Pavlov, G. G., Shibata, S., \& Tsunemi, H. 2004, ApJ, 609, 186

Pavlov, G. G., Kargaltsev, O., \& Brisken, W. F. 2008, ApJ, 675, 683

Roberts, M. S. E., Romani, R. W., Johnston, S., \& Green, A. J. 1999, ApJ, 515 712

Romani, R. W., Ng, C.-Y., Dodson, R., \& Brisken, W. 2005, ApJ, 631, 480

Slane, P., Chen, Y., Schulz, N. S., Seward, F. D., Hughes, J. P., \& Gaensler, B. M. 2000, ApJ, 533, L29

Torii, S., et al. 2008, arXiv:0809.0760

Uchiyama, H., Matsumoto, H., Tsuru, T. G., Koyama, K., \& Bamba, A. 2009, PASJ, 61, S189

van der Swaluw, E. 2003, A\&A, 404, 939

Van Etten, A., Romani, R. W., \& Ng, C.-Y. 2008, ApJ, 680, 1417

Yatsu, Y., Kawai, N., Shibata, S., \& Brinkmann, W. 2009, PASJ, 61, 129 